\title{
Analysis of genetic damage in individuals exposed to Chronic Mechanical Irritation (CMI) in the lateral border of the tongue due to Cusp of Carabelli (CoC) - A Cytogenetic study.
}

\section{Thuckanickenpalayam Ragunathan Yoithap prabhunath ${ }^{1}$, Seeni Renugadevi ${ }^{2}$, Kenniyan Kumar Sri Chinthu ${ }^{3}$, Shanmugam Mohanapriya ${ }^{4}$, Krubakar Rachel SarahVinodhini ${ }^{5}$, Swetha Pasupuleti ${ }^{6}$}

\author{
${ }^{1,2}$ Reader and Postgraduate student, Department of Oral and Maxillofacial Pathology, Vivekanandha Dental College for Women, \\ Tamilnadu, India \\ ${ }^{3}$ Reader, Department of Oral and Maxillofacial Pathology, KSR Institute of Dental Science \& Research, Tamilnadu, India \\ ${ }^{4,5}$ Consultant Oral and Maxillofacial Pathologists, Tamilnadu, India \\ ${ }^{6}$ Reader, Vishnu Dental College, Bhimavaram, Andhra Pradesh, India \\ Correspondence: yoitha.dentist@gmail.com
}

\begin{abstract}
Background: Chronic inflammation is estimated to contribute approximately $25 \%$ of human cancers. Inflammation, induced by chronic mechanical irritation (CMI) along with carcinogens has a higher chance of leading to oral pre-cancer and oral cancer. Though many studies have been conducted to identify the genetic damage in oral cancer or dysplastic stages, there is no data to find the early detection of genetic damage in healthy individuals associated with CMI. Cusp of Carabelli (CoC) is a small additional cusp at the mesiopalatal line angle of maxillary first molars which can cause chronic irritation to the lateral border of the tongue.

Objective: The study is aimed to analyze genetic damage (micronuclei and nuclear buds) in healthy individuals associated with $\mathrm{CoC}$ over the lateral border of the tongue causing CMI.

Materials and methods: The study comprised of 75 clinically healthy individuals with unilateral/bilateral presence of CoC causing irritation over the lateral border of the tongue as study group. Brush samples of lesional epithelial cells were collected, then spread over clean glass slide and fixed in 100\% alcohol, stained with rapid PAP and analyzed under the light microscope. The exfoliated cells were examined to detect the presence of micronuclei (MN).

Results: Incidence of $\mathrm{MN}$ was found to be more in $\mathrm{CoC}$ associated lateral border of the tongue of healthy individuals without habits.

Conclusions: Cytogenetic analysis is a simple and scantly invasive technique allowing clinicians in early detection of DNA damage in patients with $\mathrm{CoC}$ and preventing the carcinogenesis by proper treatment and follow-up. .
\end{abstract}

Key Words: Micronuclei, nuclear bud, Cusp of Carabelli, inflammation, chronic mechanical irritation.

\section{Introduction}

The Cusp of Carabelli (CoC), or Carabelli's tubercle, or tuberculum anomale of Georg Carabelli is a small additional cusp first described in 1842 by the Hungarian Georg Carabelli (Carabelli György), the court dentist of the Austrian Emperor Franz. It is a heritable feature found at the mesiopalatal line angle of maxillary first molars and becomes progressively less likely in the second, third molars. Though the aetiology is unknown, it is suggested that it might be due to overactivity of dental lamina or a genetic and exogenous factor seems to have an influence on formation of this cusp.

This cusp is entirely absent in some individuals and present in others in a variety of forms. In some cases, the $\mathrm{CoC}$ may rival the main cusps in size. The Carabelli's trait has been used as a critical ethnic indicator for several decades, most likely because it can be simply observed in both living individuals and skeletal remains, and can, therefore, be used to show major ethnic differences in dentition. The $\mathrm{CoC}$ is most common among Europeans (75-85\% of individuals) and rarest in Pacific Islands (35$45 \%$ ), although no study was referenced here to back up that claim.[1-3]

Patients with oral cancer often present late to the doctor making treatment difficult, expensive and sometimes unsuccessful. Delay in presentation may be considered to be health risk-taking behaviour. Chronic mechanical irritation (CMI) may not be able to produce genetic 
mutation but may prompt epigenetic changes that ultimately promotes genetic damage and inhibits DNA repair. [4] One such CMI can be due to CoC. The first documented proposition of an association between inflammation and cancer has been attributed to the German pathologist Rudolf Virchow.[5]

There are always clues right before our eyes that help us to prevent a mishap rather to treat the damage. One such clue for the development of oral cancer is identifying and analyzing the genetic damage. Unstable chromosome aberrations can be studied in the epithelial cells by the detection of micronuclei in exfoliated interphase cells. Micronuclei (MN) are small extra nuclear DNA particle formed when the chromosome fragment or acentric chromosomes fail to be included in the main nuclei of daughter cells during anaphase stage of the cell cycle. Since 1937, Micronuclei have been used as an indicator of genotoxic exposure due to their association with chromosomal aberrations. They can be detected in exfoliated cells through cytological assay which involves the microscopic analysis of epithelial smears to determine the prevalence of genetic damage, an indicator of structural or numerical chromosomal aberrations.[6-10]

With this background, this study was aimed to bring out the association of genetic damage (MN) along the lateral border of tongue in clinically healthy individuals with $\mathrm{CoC}$.

\section{Materials and methods:}

A total of 25 participants, both male and female of age group around 17 to 25 years were selected for the study. The inclusion criteria for the study was the presence of $\mathrm{CoC}$ which caused chronic irritation or ulceration on the lateral border of the tongue in relation to it. The exclusion criteria included usage of tobacco in any forms like chewing, smoking, alcoholism and any other underlying systemic conditions. Patients who were on treatment for these premalignant lesions and conditions, premalignant lesions and conditions that have progressed to malignancy and patients recently exposed to radiation or radiation therapy were excluded.

\section{Study group was subdivided into 2 groups:}

Study Group: 25 subjects having $\mathrm{CoC}$ in left posterior region causing chronic mechanical irritation in the lateral border of the tongue

Control Group: In the same study population, the right lateral border of the tongue, which didn't have $\mathrm{CoC}$ was considered as control.

Sample collection: A cyto-brush was used to scrape the CoC-A chronic mechanical irritant? exfoliated cells along the lateral borders of the tongue (both left and right). Before the collection of smears, the subjects were asked to rinse their mouth with tap water. The collected smear was then spread over clean glass slide and fixed in $100 \%$ alcohol. Before staining, the fixed smears were washed in running tap water to remove bacteria and debris which may interfere with the scoring.

Staining and evaluation of the slides: Rapid Papanicolaou (PAP) staining kit was used to stain the slides containing the fixed smears. Slides were evaluated using Tolbert et al,[11] criteria for classification of nuclear abnormalities. The cells were counted under 40x magnification. A total of 100 cells per subject were counted. Cells with indefinite borders, overlapping cells were excluded from counting. The MN present in each cell was then determined. The samples were analyzed and photomicrographs were taken using Leica DMD 108 micro-imaging system [Figure 1].

Statistical analysis: Software used was Statistical package for social science SPSS version 16 (IBM CORP, Chicago, IL, USA). The level of significance $(p<0.05)$ was employed in all statistical comparisons. Quantitative data were recorded as mean \pm standard deviation. Data were subjected to paired and unpaired t-tests.

\section{Ethics}

The study was carried out after getting the proper permission from the Institutional Ethical Committee (VDCW/IEC/57/2017) and after getting the consent from the study participants.

\section{Results-}

A total number of 100 cells were counted for the determination of micronuclei from lateral borders of tongue on both sides of the study population as control group and study group containing $\mathrm{CoC}$. On statistical analysis it was found that the mean value for $\mathrm{MN}$ count in $\mathrm{CoC}$ associated left lateral border of tongue (169.25) was higher than the normal tooth associated side (140.43) [Table 1] and was found to be statistically significant.

\section{Discussion}

MNs are fragments that occurs as a result of failure of fragments or whole chromosomes that lag behind at anaphase during nuclear division. They are widely used as cytological biomarkers for the evaluation genetic damage due to exposure of genotoxins and carcinogens and manifestation of which can act as cancer risk biomarkers. Several micronuclei assay studies in the past have brought out the association of micronuclei in genotoxic group, thus 


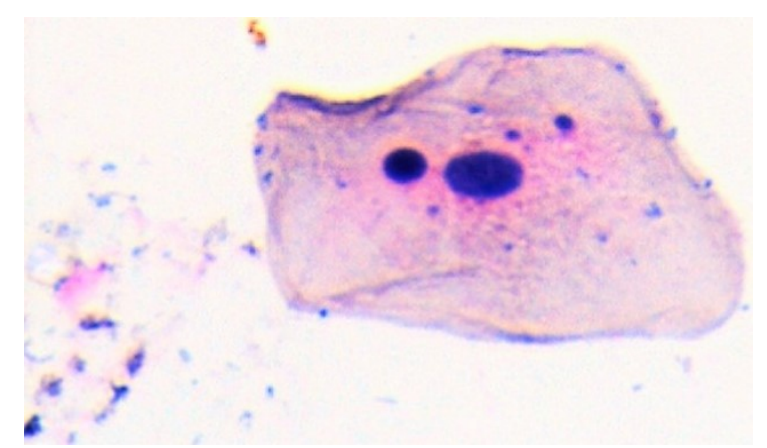

Fig -1 Exfolliated cells showing Micronuclei.

supporting the use of micronuclei assay as a potent biomarker for assessing DNA damage. [6-10]

Previous studies have stated the hypothesis that, CMI could act at least as a co-factor in oral carcinogenesis and it is underrated as a potential risk factor. Tongue is the most common site for Sustained traumatic ulcers (Decubitus ulcer) and Traumatic ulcerative granuloma (Eosinophilic ulcer of the tongue). Traumatic ulcers heal within 7 to 10 days but some persist for weeks to months due to constant irritation.[13] The lateral border of tongue was found to be the most common site of tumour occurrence in both smokers and nonsmokers. The incidence of tumour occurrence in lateral border of tongue was twice as common in nonsmokers when compared to smokers. $\mathrm{CMI}$ due to $\mathrm{CoC}$ mainly cause irritation in the lateral border of the tongue which results in genetic instability. Carcinogens which come in contact with these genetically damaged areas have a higher chance of resulting in mutation. These CMI induced damage in lateral border of tongue could be a most frequent site of chronic trauma associated oral cancer.[14]

In the present study, cytological smear from the $\mathrm{CoC}$ associated with lateral border of the tongue revealed higher incidence of genetic damages. Micronuclei count increased in the affected side comparing to that of the normal side. Results from the study states a strong relationship between CMI due to presence of $\mathrm{CoC}$ in relation to lateral border of the tongue.

An experimental study in hamsters has shown that chronic trauma in addition to carcinogen application could promote tumour initiation. In this study, mechanical irritation by scratching with a pulp cleaner has been shown to significantly increase the incidence of a chemical carcinogen-induced tongue carcinoma. Therefore, it is prudent to closely monitor patients with known risk factors for signs and symptoms of irritation from teeth (Sharp/broken teeth) [15].

CoC - A chronic mechanical irritant?
Inflammation is a perilous component of tumour progression. Past studies state that many cancers arise from sites of infection, chronic irritation and inflammation. It also states that for promoting proliferation, survival and migration of tumour cell, tumour microenvironment, which is largely orchestrated by inflammatory cells, is essential.[14] In 1863, Virchow hypothesized that the origin of cancer was at sites of chronic inflammation. In part based on his hypothesis that some classes of irritants, together with the tissue injury and ensuing inflammation they cause, enhance cell proliferation.[5] Under an inflammatory microenvironment, exposure to Reactive Oxygen Species / Reactive Nitrogen Species (ROS/ RNS) result in genomic instability. Multistage carcinogenesis consists of three steps: tumour initiation, promotion, and progression. The genomic instability or damage will result in tumour initiation.[15]

Casartelli et al, (2000) observed micronuclei frequencies in exfoliated buccal cells in normal mucosa, precancerous lesion and squamous cell carcinoma. Thus, they have arrived at a conclusion stating that a gradual increase in micronuclei count from normal mucosa to precancerous to carcinoma thereby suggesting a link of this biomarker with neoplastic progression. The initiation and progression of cancer has now been largely attributed to the role played by inflammatory cells like cytokines and growth factors like chemokines. [15,16]

In the present study, the results revealed a significant increase in the incidence of micronuclei on the lateral border of the tongue where the presence of $\mathrm{CoC}$ caused CMI. On comparing the case and the control group significant difference exists, stating that inflammation could be an important co factor, where the initiation process of carcinogenesis can be induced along with the exposure to carcinogenic agent.

Among the various genetic studies, micronuclei assay plays an important role in predicting the various diseases. It found to be the non-invasive method of detecting the 
malignant cells in diagnosis and treatment.

\section{Conclusion}

Many studies on micronucleus assay has been done which includes the study of micronuclei in the exfoliated oral epithelial cells in individuals with different parameters like CMI, smoking, consumption of alcohol, use of betel nut and tobacco etc. But till date $\mathrm{CoC}$ was considered just as a trait and not as CMI. The definitive evidence for the same is lacking. We undertook a search using the terms - CoC, dental trauma, mucosal trauma, oral cancer, squamous cell carcinoma, risk factor, genetic damage, micronuclei, nuclear bud, potentially malignant lesion, dental factor, mechanical irritation, dental irritation, and cancer in the following electronic databases: MEDLINE, PubMed, ScienceDirect, and Wiley InterScience. To the best of our knowledge, this was the very first study made to analyze the early association of $\mathrm{CoC}$ with the incidence of micronuclei.

\section{Limitation}

Increased sample size could be considered in the future along with cytokinesis-block cytogenetic analysis. Nuclear budding (NB), and Nucleoplasmic bridge (NPB) can also be added as a criterion to evaluate the genetic damage caused by CMI due to CoC. Long term follow-up is necessary to find the association of sharp tooth induced CMI and dysplasia/oral cancer in lateral border of the tongue.

\section{References}

1. Kirthiga M, Manju M, Praveen R, Umesh W. Ethnic Association of CoC Trait and Shoveling Trait in an Indian Population. J Clin Diagn Res. 2016;10(3):ZC78 $-\mathrm{ZC} 8$.

2. Smitha T, Venkatesh $\mathrm{D}$, Veeresh $\mathrm{M}$, Hema $\mathrm{K} \mathrm{N}$, Sheethal H S, Vidya M A. The CoC: Frequency, distribution and type in the Bengaluru population. J Oral Maxillofac Pathol 2018;22:418-22.

3. Falomo OO. The CoC: Frequency, distribution, size and clinical significance in Nigeria. West Afr J Med. 2002;21:322-24.

4. Lazos JP, Piemonte ED, Lanfranchi HD, Brunotto MN. Characterization of Chronic Mechanical Irritation in Oral Cancer. International Journal of Dentistry. 2017, Article ID 6784526, 7.

5. Lu, H. (2006). Inflammation, a key event in cancer development. Molecular Cancer Research, 4(4), pp.221-233.

6. Fenech M, Kirsch-Volders M, Natarajan AT, Surralles J, Crott JW, Parry J, Norppa H, Eastmond DA, Tucker

CoC - A chronic mechanical irritant?
JD, Thomas P. Molecular mechanisms of micronucleus, nucleoplasmic bridge and nuclear bud formation in mammalian and human cells. Mutagenesis. 2011 Jan;26(1):125-32.

7. Cheong HS, Seth I, Joiner MC, Tucker JD. Relationships among micronuclei, nucleoplasmic bridges and nuclear buds within individual cells in the cytokinesis-block micronucleus assay. Mutagenesis. 2013 Jul;28(4):433-40.

8. Gashi G, Mahovlić V, Manxhuka-Kerliu S, Podrimaj -Bytyqi A, Gashi L, Elezaj I. The association between micronucleus, nucleoplasmic bridges, and nuclear buds frequency and the degree of uterine cervical lesions. Biomarkers. 2018;23(4):364-372.

9. Gupta A, Bhovi TV, Jaju PP, GuptaA, Gupta M, Shrivastava K. Micronuclei frequency as an early diagnostic tool for detection of Oral Cancer: A comparative study. International Journal of Oral Health Dentistry; April-June 2016;2(2):77-83.

10. Kohli M, Ahuja P, Mehendiratta M, Sharma M, Dutta J. Micronucleus Assay: An Early Diagnostic Tool to Assess Genotoxic Changes in Patients with Tobacco Use, Oral Leukoplakia and Oral Submucous Fibrosis. J Clin Diagn Res. 2017;11(9):ZC28-ZC32.

11. Tolbert PE, Shy CM, Allen JW. Micronuclei and other nuclear anomalies in buccal smears: methods development. Mutat Res. 1992 Feb;271(1):69-77.

12. Singhvi HR, Malik A, Chaturvedi P. The Role of Chronic Mucosal Trauma in Oral Cancer: A Review of Literature. Indian J Med Paediatr Oncol. 2017;38 (1):44-50.

13. Kumar M, Nanavati R, Modi TG, Dobariya C. Oral cancer: Etiology and risk factors: A review. J Can Res Ther 2016;12:458 $\square 63$.

14. Coussens LM, Werb Z. Inflammation and cancer. Nature. 2002;420(6917):860-7.

15. Singhvi HR, Malik A, Chaturvedi P. The role of chronic mucosal trauma in oral cancer: A review of literature. Indian J Med Paediatr Oncol 2017;38:44-50.

16. Casartelli G, Bonatti S, De Ferrari M, Scala M, Mereu P, Margarino G, Abbondandolo A. Micronucleus frequencies in exfoliated buccal cells in normal mucosa, precancerous lesions and squamous cell carcinoma. Anal Quant Cytol Histol. 2000 Dec;22 (6):486-92.

How to cite: Yoithap prabhunath TR, Renugadevi S, Sri chinthu KK, Mohanapriya S, Rachel Sarah Vinodhini K, Swetha $P$.Analysis of genetic damage in individuals exposed to Chronic Mechanical Irritation (CMI) in the lateral border of the tongue due to Cusp of Carabelli $(\mathrm{CoC})-A$ Cytogenetic study. Ind J Clin Dent 2020;1(1):1-4 